\title{
Yoga for chronic chemotherapy-induced peripheral neuropathy pain: a pilot, randomized controlled trial
}

\author{
Robert Knoerl ${ }^{1,2}$ (D) Anita Giobbie-Hurder ${ }^{3} \cdot$ Juliana Berfield $^{4} \cdot$ Donna Berry $^{5} \cdot$ Jeffrey A. Meyerhardt $^{6}$. \\ Alexi A. Wright ${ }^{6}$. Jennifer A. Ligibel $^{6}$
}

Received: 4 May 2021 / Accepted: 29 June 2021 / Published online: 15 September 2021

(c) The Author(s), under exclusive licence to Springer Science+Business Media, LLC, part of Springer Nature 2021

\begin{abstract}
Purpose To determine the feasibility of implementing a yoga intervention for cancer survivors with chronic CIPN pain, as well as the impact of the intervention on patient-reported outcomes.

Methods Cancer survivors with chronic CIPN pain were recruited from the breast, gastrointestinal, and gynecological oncology centers at Dana-Farber Cancer Institute. Participants were randomized (2:1) to receive an 8-week yoga intervention or usual care. After 21/50 of participants were enrolled, the COVID-19 pandemic required the yoga intervention to be delivered virtually (i.e., Zoom). Pre- and post-intervention, participants self-reported CIPN and co-occurring symptom severity. Adherence to the intervention was defined as practicing $\geq 12$ yoga sessions over the 8 -week intervention period. Changes in patient-reported outcomes between groups were compared using Wilcoxon's rank-sum tests.

Results Participants ( $n=28$ yoga, $n=16$ control) were mainly female $(96 \%)$ and diagnosed with stage III/IV disease (66\%). Overall, 19/28 (67.8\%) of yoga group participants were adherent to the yoga protocol. Yoga group participants experienced significant within-group improvements in all patient-reported outcomes, including worst CIPN pain (median change $=-1.7$, $p<0.0001$ ) and sensory CIPN (median change $=-14.8, p<0.0001$ ), but only improvements in fatigue $(p=0.05)$ and depression $(p=0.04)$ were significant compared to the control. There were no differences $(p>0.05)$ in changes in patient-reported outcomes between in-person $(n=6)$ or virtual $(n=15)$ yoga group participants.

Conclusions Yoga is a feasible non-pharmacological modality for cancer survivors with CIPN, but more information is needed regarding its impact on CIPN and other symptoms.

Trial registration ClinicalTrials.gov Identifier: NCT03824860

Implications for Cancer Survivors Oncology clinicians may consider referring cancer survivors to yoga for chronic CIPN pain, but yoga cannot be currently recommended as an efficacious treatment.
\end{abstract}

Keywords Yoga $\cdot$ Chemotherapy-induced peripheral neuropathy $\cdot$ Neoplasms $\cdot$ Randomized controlled trial [publication type] $\cdot$ Cancer survivors $\cdot$ Integrative oncology

Robert Knoerl

Robert_knoerl@dfci.harvard.edu; rjknoerl@med.umich.edu

1 Phyllis F. Cantor Center for Research in Nursing and Patient Care Services, Dana-Farber Cancer Institute, 450 Brookline Ave, LW 518, Boston, MA 02215, USA

2 Present Address: University of Michigan School of Nursing, 400 North Ingalls St, Office 2350, Ann Arbor, MI 48109, USA

3 Division of Biostatistics, Department of Data Science, Dana-Farber Cancer Institute, Boston, MA, USA
4 The Leonard P. Zakim Center for Integrative Therapies and Healthy Living, Dana-Farber Cancer Institute, Boston, MA, USA

5 Biobehavioral Nursing and Health Informatics, University of Washington, Seattle, WA, USA

6 Department of Medical Oncology, Dana-Farber Cancer Institute, Boston, MA, USA 


\section{Introduction}

Chemotherapy-induced peripheral neuropathy (CIPN), which commonly manifests as numbness, tingling, and pain in the hands or feet, is one of the most common sources of long-term morbidity in cancer patients treated with chemotherapy. Approximately $35 \%$ of patients experience painful CIPN symptoms for more than 3 months after the completion of neurotoxic chemotherapy treatment (e.g., taxanes or platinums) [1], impairing physical function long after treatment completion [2]. Functional impairments related to CIPN are associated with an increased risk of falls [3], difficulty completing daily activities [4], and an inability to return to work [5]. In addition, painful CIPN symptoms may cluster together with symptoms such as sleep disturbance, anxiety, fatigue, and depression $[6,7]$.

Although the negative effects of CIPN are well documented, there remains an urgent need for the development and identification of efficacious treatments for CIPN. The American Society of Clinical Oncology concluded that only duloxetine $60 \mathrm{mg} /$ day [8] can be recommended for painful CIPN [9]. However, evidence suggests that duloxetine is infrequently prescribed for painful CIPN [10] due to difficulties such as securing insurance payment [11]. Cancer survivors with CIPN are twice as likely to be prescribed opioids than those without CIPN [12], despite the lack of data supporting the use of opioids for this condition. Therefore, investigation of novel, non-pharmacological treatments for CIPN is warranted.

Yoga is a mind-body intervention that incorporates physical postures, breathing, and meditation to increase flexibility and strength, relaxation, and body awareness [13, 14]. Yoga's focus on improving strength and balance is applicable to cancer survivors with chronic CIPN pain because such individuals may be at an increased risk of fall due to lower extremity sensory or motor CIPN symptoms. Yoga has also been shown to improve cancer treatment-related symptoms such as fatigue and sleep disturbance [15], symptoms that are known to cluster with painful CIPN [6,7].

The primary purpose of this pilot study was to determine the feasibility of implementing an 8-week yoga intervention in cancer patients with chronic CIPN pain by evaluating participant recruitment, retention, and adherence rates. A secondary aim was to characterize withinand between-group changes over the 8-week intervention period with regard to worst CIPN pain intensity, CIPN severity, physical function, sleep-related impairment, anxiety, depression, and fatigue among cancer survivors with chronic CIPN pain who received the yoga intervention or treatment as usual.

\section{Methods}

\section{Design, setting, and sample}

A parallel, randomized controlled trial was employed to investigate the study aims. Patients with chronic CIPN pain were recruited from the breast, gastrointestinal, and gynecological oncology centers at the Dana-Farber Cancer Institute. Participants were eligible if they were Englishspeaking adults (i.e., $\geq 18$ years old) diagnosed with stage I-IV breast, colorectal, or gynecologic cancer, and at least 3 months post-taxane or platinum-based chemotherapy with a self-reported $\geq 4 / 10$ worst CIPN pain over the past week [16]. Participants were ineligible if they had a prognosis of less than 3 months, had documented neuropathy due to other causes (e.g., diabetes), planned to receive ongoing neurotoxic chemotherapy during the study, had a diagnosis or documented history of significant psychiatric comorbidity (i.e., psychosis, suicidal ideation, or substance abuse) [16], or practiced yoga an average of $>45$ min per week over the past 6 months. The study was approved by the Dana-Farber/ Harvard Cancer Center Institutional Review Board.

\section{Treatment groups}

Yoga Participants randomized to the intervention arm were treated with an 8-week yoga program consisting of therapist-guided and self-guided yoga components. Participants attended in-person yoga sessions offered through the Leonard P. Zakim Center for Integrative Therapies and Healthy Living at Dana-Farber (Zakim Center) and had access to prerecorded yoga sessions developed for the study by a yoga therapist (JB) at the Zakim Center. Participants received an initial one-on-one in-person meeting with a yoga therapist to review proper form and goals for yoga participation before beginning the intervention. Yoga sessions were $45 \mathrm{~min}$ in length, were taught by a certified yoga therapist, and consisted of guided breathing exercises, upper and lower extremity stretching, and structured postures and movements to improve balance and strength (Online Resource 1). For the self-guided component, the study team recorded yoga sessions and a segment about hand and foot stretches. All videos were made available to participants electronically via email. All yoga group participants received yoga balls and blocks to facilitate at-home practice. To enhance adherence to the intervention, study staff made weekly calls to participants to discuss yoga practice and participants received free parking vouchers when attending yoga sessions at the Zakim Center.

After 21 participants were enrolled, the Zakim Center closed to in-person sessions due to the COVID-19 pandemic. 
Following the closure, the initial session with the yoga therapist and all yoga sessions were offered via livestreamed sessions on Zoom. Participants were considered to have attended an "in-person" yoga class if they attended the livestreamed Zoom class. During the pandemic, participants still received weekly calls from study staff to discuss yoga practice, but the yoga instructor could not directly observe participants' practice as yoga sessions were provided as webinars to preserve patient privacy.

Wait-list control Control group participants received usual care and symptom management strategies through their typical oncology provider during the study. Control group participants did not receive any study treatment or intervention. After the 8-week study period, the control group participants were encouraged to attend the yoga sessions offered by the Zakim Center and were provided with access to the virtual yoga videos.

\section{Measures}

Feasibility Feasibility was defined a priori as (1) recruitment over 1.5 years ( $\sim 3$ recruited per month), (2) completion of baseline and end of study outcome measures by $\geq 60 \%$ of participants, and (3) attendance at $\geq 12$ in-person (or live Zoom sessions during the COVID-19 pandemic) or selfguided/at-home yoga sessions during the 8-week study period by $\geq 60 \%$ of participants. An at-home session was defined as yoga practice of at least $15 \mathrm{~min}$ or more over the course of a week. Yoga group participants completed a weekly exercise log to collect information about the frequency of yoga practice, other exercise and symptom management strategies, and study-related adverse events.

Patient-Reported Outcome Measures Participants selfreported worst CIPN pain intensity for seven consecutive days using a $0-10$ numerical rating scale of pain intensity $[17,18]$. Several Patient-Reported Outcome Measurement Information System (PROMIS®) short-form instruments [19] were also used to measure pain interference (PROMIS ${ }^{\circledR}$ Pain Interference 4a) [20], sleep-related impairment (PROMIS ${ }^{\circledR}$ Sleep-related Impairment 8a) [21], anxiety (PROMIS® Anxiety 4a) [22], fatigue (PROMIS® Fatigue 4a) [23], depression (PROMIS® Depression 4a) [22], and physical function (PROMIS® Physical Function 4a) [24]. Higher scores on all measures except the Physical Function 4a represented worse symptom severity. CIPN severity was measured using the sensory and motor subscales of the European Organization of Research and Treatment of Cancer Quality of Life Questionnaire-Chemotherapy-Induced Peripheral Neuropathy (QLQCIPN20) [25]. The QLQ-CIPN20 is a twenty-item instrument that measures self-reported severity and functional limitations in sensory (e.g., numbness, tingling, pain), motor (e.g., extremity weakness), and autonomic (e.g., dizziness) domains. Each subscale is scored on a transformed score of $0-100$ (worse symptoms) [25]. Both the sensory $(\alpha=0.88)$ and motor $(\alpha=0.88)$ subscales have demonstrated sufficient internal consistency reliability [26]. Evidence supports the divergent validity of the sensory and motor subscales based on their ability to discriminate neuropathy severity between participants who had and who had not received neurotoxic chemotherapy [26]. The autonomic subscale was not administered because the subscale demonstrates poor psychometric properties [26, 27].

\section{Procedures}

Participants completed the demographic questionnaire, pain intensity diary, PROMIS ${ }^{\circledR}$ measures, and the QLQ-CIPN20 within 10 days after consent. After completing the baseline measures, participants were randomized in a $2: 1$ ratio using a computer-generated random numbers table. The randomization was stratified according to baseline pain intensity diary scores $(<7$ or $\geq 7)$. A statistician (AGH) generated the random numbers table. Throughout the 8-week study period, yoga group participants completed the weekly exercise log. After the study period, participants completed the pain intensity diary, PROMIS ${ }^{\circledR}$ measures, and QLQ-CIPN20. Study staff also abstracted information regarding participants' cancer type and stage, chemotherapy, and comorbid conditions from the medical record.

\section{Statistical analysis}

The primary aim of this pilot study was feasibility. The observed recruitment, retention, and adherence rates were estimated along with two-sided $80 \%$ exact confidence intervals (CIs). For the secondary aims, estimates of changes in patient-reported outcomes were calculated using an intentto-treat approach. Demographic and patient-reported outcomes were summarized with descriptive statistics. Within each treatment arm, the paired change in outcome scores from baseline to the end of study was compared with zero using Wilcoxon's signed-rank tests, and the difference in the paired change in scores between treatment arms was compared using Wilcoxon's rank-sum tests. Due to the switch in intervention format during the study, comparisons of changes in patient-reported outcomes between the yoga intervention formats were also explored using Wilcoxon's rank-sum tests. Statistical significance was defined as $p \leq 0.05$ and there were no adjustments for multiple comparisons.

Sample size considerations With a target sample size of 50 participants, the exact $80 \%$ confidence interval (CI) for the rate of study completion would have been no wider than 
19.9\%. Further, with approximately 33 participants randomized to the yoga intervention, the exact $80 \%$ (CI) for adherence measures would have been no wider than $24.7 \%$. For assessment of changes between the randomized groups during the 8-week intervention, a Wilcoxon rank-sum test with two-sided, 0.1 type I error had $80 \%$ power to detect a difference in change scores that is 0.80 times the common standard deviation.

\section{Results}

\section{Sample characteristics}

Figure 1 displays the study flow diagram [28]. Overall, 50 participants were consented, and 45 were randomized (yoga $n=29$, control $n=16$ ). Five participants did not complete baseline study measures and were lost to followup between registration and randomization. One patient was found to be ineligible after randomization, leaving a total sample size of 44 patients (yoga $n=28$, control $n=16$ ). The reduction in sample size increased the maximum confidence interval widths for study completion and intervention compliance to $21.2 \%$ and $27.0 \%$, respectively, and the detectable effect size between randomized groups to 0.835 . Table 1 describes the demographic characteristics of the 44 eligible randomized participants. Participants' demographic characteristics and patient-reported symptom severity were generally well balanced between the randomized arms, but yoga group participants had significantly higher depression severity than control group participants $(p=0.007)$ at baseline.
Fig. 1 Consort flow diagram. The figure describes yoga and control group participants' progress through the study

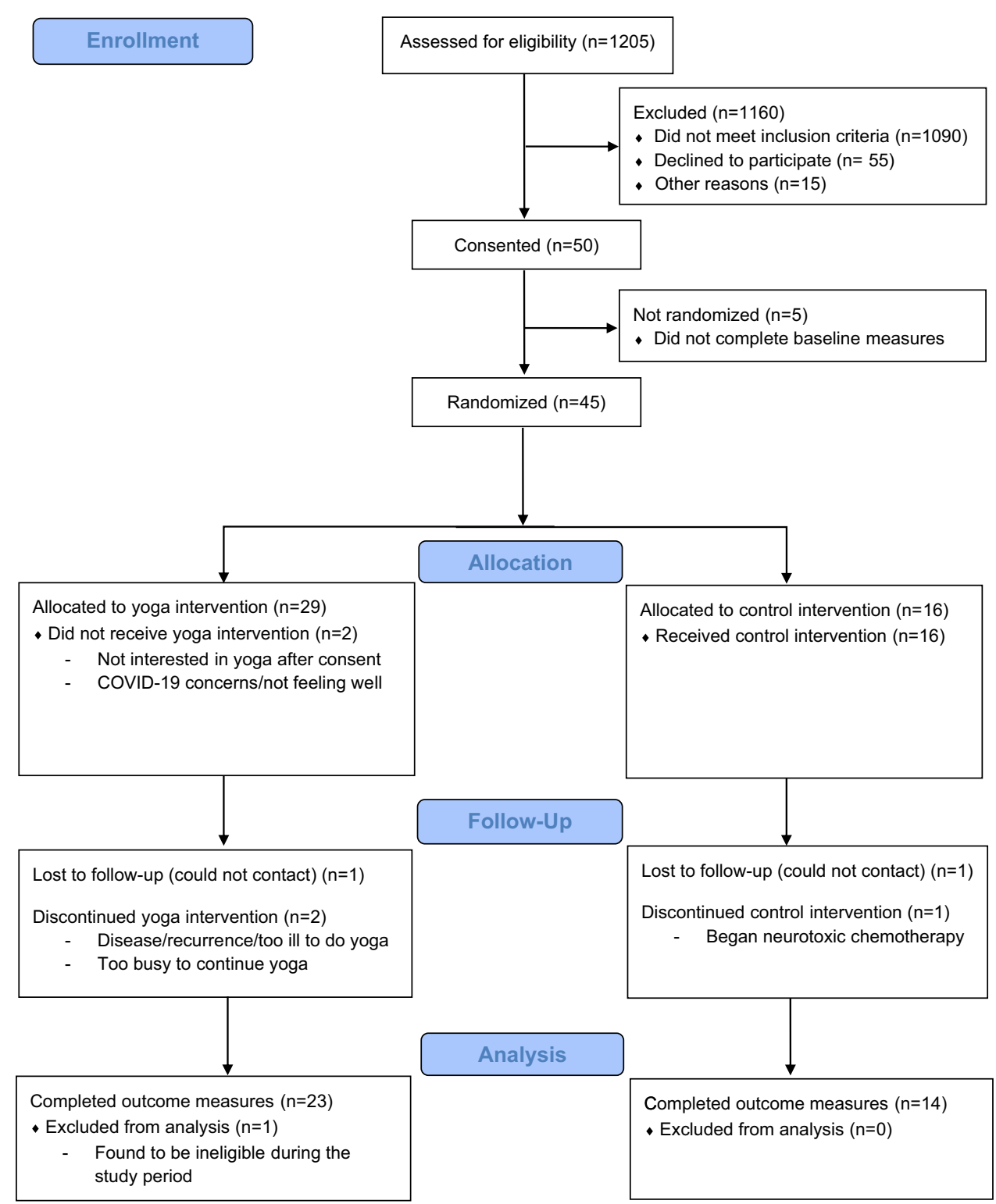


Table 1 Baseline demographic characteristics of participants randomized to the yoga or control groups

\begin{tabular}{|c|c|c|}
\hline Characteristic & Yoga $(n=28)$ & Control $(n=16)$ \\
\hline \multicolumn{3}{|l|}{ Age } \\
\hline Median (range) & $60(33-74)$ & $56.5(40-79.0)$ \\
\hline \multicolumn{3}{|l|}{ Sex } \\
\hline Female & $27(96.4 \%)$ & $15(93.8 \%)$ \\
\hline Male & $1(3.6 \%)$ & $1(6.3 \%)$ \\
\hline \multicolumn{3}{|l|}{ Race } \\
\hline Missing & $1(3.6)$ & 0 \\
\hline Asian & $1(3.6 \%)$ & $1(6.3 \%)$ \\
\hline Black or African American & $3(10.7 \%)$ & $2(12.5 \%)$ \\
\hline White & $22(78.6 \%)$ & $13(81.3 \%)$ \\
\hline Unknown or do not wish to report & $1(3.6 \%)$ & 0 \\
\hline \multicolumn{3}{|l|}{ Ethnicity } \\
\hline Missing & $1(3.6)$ & 0 \\
\hline Hispanic or Latino & $2(7.1 \%)$ & 0 \\
\hline Not Hispanic or Latino & $25(89.3 \%)$ & $16(100 \%)$ \\
\hline \multicolumn{3}{|l|}{ Cancer type } \\
\hline Breast & $9(32.1 \%)$ & $5(31.3 \%)$ \\
\hline Gastrointestinal $^{\mathrm{a}}$ & $7(25 \%)$ & $3(18.8 \%)$ \\
\hline Gynecological $^{\mathrm{b}}$ & $10(35.7 \%)$ & $8(50 \%)$ \\
\hline Multiple & $2(7.1 \%)$ & 0 \\
\hline \multicolumn{3}{|l|}{ Cancer stage } \\
\hline Stage I & $4(14.3 \%)$ & $2(12.5 \%)$ \\
\hline Stage II & $5(17.9 \%)$ & $3(18.8 \%)$ \\
\hline Stage III & $10(35.7 \%)$ & $9(56.3 \%)$ \\
\hline Metastatic & $8(28.6 \%)$ & $2(12.5 \%)$ \\
\hline Unknown or missing & $1(3.6 \%)$ & 0 \\
\hline \multicolumn{3}{|l|}{ Chemotherapy type } \\
\hline Oxaliplatin & $7(25 \%)$ & $3(18.8 \%)$ \\
\hline Taxanes & $9(32.1 \%)$ & $4(25 \%)$ \\
\hline Taxanes and platinums & $12(42.9 \%)$ & $9(56.2 \%)$ \\
\hline \multicolumn{3}{|c|}{ Time since last neurotoxic chemotherapy infusion } \\
\hline Median (range) & $242.5(92-1423)$ & $249.5(105-1708)$ \\
\hline \multicolumn{3}{|l|}{ Risk factors for CIPN } \\
\hline No & $6(21.4 \%)$ & $6(37.5 \%)$ \\
\hline $\mathrm{Yes}^{\mathrm{c}}$ & $22(78.6 \%)$ & $10(62.5 \%)$ \\
\hline \multicolumn{3}{|c|}{ Number of minutes of yoga in the last 7 days at baseline } \\
\hline Median (range) & $0(0-30)$ & 0 (no range) \\
\hline
\end{tabular}

Note:

${ }^{a}$ Gastrointestinal malignancies included colorectal, pancreas, gastroesophageal junction, small bowel, and extrahepatic bile duct

${ }^{\mathrm{b}}$ Gynecological malignancies included ovarian, endometrium, uterine, and fallopian tube

${ }^{\mathrm{c}}$ The most frequent potential risk factors for CIPN included anxiety, arthritis, depression, and obesity

\section{Feasibility of study recruitment and adherence to the yoga intervention}

Participant recruitment occurred from August 6, 2019, to November 17, 2020. Accrual was paused from March 11, 2020, to May 31, 2020, due to the COVID-19 pandemic, resulting in an overall accrual rate of 3.45 participants per month (44/12.7). Eighteen participants were enrolled during the 7.2 months prior to the COVID-19-related recruitment pause (2.5 participants per month) and 26 patients in the 5.6 months following the recruitment pause (4.6 participants per month). Reasons for declining study participation included commuting (pre-COVID) and lack of interest in yoga/virtual yoga (during COVID). 
Thirty-five of 44 participants (79.5\%, 80\% CI: 70 to $87 \%)$ completed baseline and end of study measures, and 19/28 participants $(67.8 \%, 80 \%$ CI: 54 to $80 \%)$ randomized to the yoga arm completed $\geq 12$ yoga sessions. Yoga participants completed an average of 15 sessions $(S D=11$, range $=0-38)$ over the 8-week study period. Prior to the COVID-19 pandemic, 33\% (3/9) of participants randomized to the yoga group completed $\geq 12$ sessions, whereas $87.5 \%$ (14/16) of participants completed $\geq 12$ sessions during the pandemic. Three yoga group participants were on study when the Zakim Center closed due to the pandemic and were instructed to practice the self-guided components only until the livestreamed sessions became available. Adverse events reported by participants that may have been related to yoga included foot pain, Achilles tendonitis, and back pain.

\section{Impact of yoga on Patient-Reported Outcome Measures}

Table 2 describes the changes in CIPN and co-occurring symptoms over the 8 -week intervention period (yoga $n=23$, control $n=14$ ). Yoga group participants

Table 2 Median (range) scores for secondary outcomes by treatment group (yoga $N=23$, control $N=14$ )

\begin{tabular}{|c|c|c|c|c|c|}
\hline \multirow[t]{2}{*}{ Outcomes } & \multicolumn{2}{|l|}{ Median $(\text { range })^{\mathrm{a}}$} & \multicolumn{2}{|l|}{ Estimate of median change (range, $p$ ) } & \multirow{2}{*}{$\begin{array}{l}\text { Comparison of } \\
\text { change between } \\
\text { groups }(p)\end{array}$} \\
\hline & Yoga & Control & Yoga & Control & \\
\hline \multicolumn{6}{|c|}{ Worst CIPN pain ${ }^{\mathrm{b}}$} \\
\hline Baseline & $5.9(3.0-9.1)$ & $5.9(3.6-8.1)^{\mathrm{d}}$ & \multirow[t]{2}{*}{$-1.7(-7.1 \text { to } 0.6, p<0.0001)^{*}$} & \multirow[t]{2}{*}{$-1.6(-4.1 \text { to } 2.0, p=0.04)^{\mathrm{d} *}$} & \multirow[t]{2}{*}{0.74} \\
\hline Eight weeks & $4.0(0-8.1)$ & $4.2(1.0-9.0)^{\mathrm{d}}$ & & & \\
\hline \multicolumn{6}{|l|}{ CIPN sensory ${ }^{\mathrm{b}}$} \\
\hline Baseline & $48.1(14.8-85.2)$ & $42.6(22.2-81.5)$ & \multirow{2}{*}{$\begin{array}{c}-14.8(-40.7 \text { to } 11.1 \\
p<0.0001)^{*}\end{array}$} & \multirow{2}{*}{$\begin{array}{l}-11.1(-40.7 \text { to } 11.1 \\
p=0.003)^{*}\end{array}$} & \multirow[t]{2}{*}{0.42} \\
\hline Eight weeks & $37.0(7.4-88.9)$ & $31.5(7.4-66.7)$ & & & \\
\hline \multicolumn{6}{|l|}{ CIPN motor ${ }^{\mathrm{b}}$} \\
\hline Baseline & $33.3(0-91.7)$ & $25.0(4.2-66.7)$ & \multirow{2}{*}{$\begin{array}{c}-8.3(-45.8 \text { to } 33.3 \\
p=0.0004)^{*}\end{array}$} & \multirow{2}{*}{$-8.3(-29.2 \text { to } 8.3, p=0.002)^{*}$} & \multirow[t]{2}{*}{0.73} \\
\hline Eight weeks & $25.0(0-58.3)$ & $16.7(0-37.5)$ & & & \\
\hline \multicolumn{6}{|c|}{ Pain interference ${ }^{\mathrm{b}}$} \\
\hline Baseline & $59.9(41.6-75.6)$ & $59.2(52-66.6)$ & \multirow[t]{2}{*}{$-4.6(-22.2 \text { to } 11.2, p=0.002)^{*}$} & \multirow[t]{2}{*}{$-4.2(-18.3 \text { to } 5.6, p=0.03)^{*}$} & \multirow[t]{2}{*}{0.99} \\
\hline Eight weeks & $53.9(41.6-69.7)$ & $54.8(41.6-71.6)$ & & & \\
\hline \multicolumn{6}{|c|}{ Physical function ${ }^{c}$} \\
\hline Baseline & $40.5(33.2-57)$ & $42.7(30.5-48.3)$ & \multirow[t]{2}{*}{$3.5(-7.6 \text { to } 19.1, p=0.003)^{*}$} & \multirow[t]{2}{*}{$1.7(-11.6$ to $11.6, p=0.22)$} & \multirow[t]{2}{*}{0.49} \\
\hline Eight weeks & $45.5(31.9-57)$ & $44.5(31.9-57)$ & & & \\
\hline \multicolumn{6}{|l|}{ Anxiety $^{b}$} \\
\hline Baseline & $57.7(40.3-71.2)$ & $53.5(40.3-71.2)$ & \multirow{2}{*}{$\begin{array}{c}-5.6(-23.1 \text { to } 10.2 \\
p=0.0007)^{*}\end{array}$} & \multirow{2}{*}{$0(-19.2$ to $15.5, p=0.65)$} & \multirow[t]{2}{*}{0.13} \\
\hline Eight weeks & $51.2(40.3-71.2)$ & $51.2(40.3-71.2)$ & & & \\
\hline \multicolumn{6}{|l|}{ Depression $^{\mathrm{b}}$} \\
\hline Baseline & $53.9(41.0-63.9)$ & $45.0(41.0-53.9)$ & \multirow[t]{2}{*}{$0(-12.9 \text { to } 4.9, p=0.01)^{*}$} & \multirow[t]{2}{*}{$0(-10.8$ to $19.5, p=0.50)$} & \multirow[t]{2}{*}{$0.04 *$} \\
\hline Eight weeks & $49.0(41.0-63.9)$ & $49.0(41.0-60.5)$ & & & \\
\hline Sleep impairmeı & & & & & \\
\hline Baseline & $59.3(35.2-66.3)$ & $55.1(35.2-71.9)$ & $-5.0(-25.1 \text { to } 24.6, p=0.02)^{*}$ & $-1.2(-10.0$ to $10.4, p=0.79)$ & 0.10 \\
\hline Eight weeks & $50.3(30.0-69.5)$ & $54.8(38.7-69.5)$ & & & \\
\hline Fatigue $^{b}$ & & & & & \\
\hline Baseline & $55.1(33.7-75.8)$ & $53.1(43.1-71.6)$ & $-6.5(-14.9 \text { to } 21.4, p=0.002)^{*}$ & $-1.4(-13.6$ to $9.6, p=0.42)$ & $0.05 *$ \\
\hline Eight weeks & $48.6(33.7-66.7)$ & $53.1(43.1-71.6)$ & & & \\
\hline
\end{tabular}

Note:

${ }^{a}$ Increasing scores for the specified variables indicate worse symptoms, except for physical function, where higher scores represent greater function

${ }^{\mathrm{b}}$ For the estimate of median change, negative estimates indicate improvements in symptom severity, whereas positive estimates indicate worsening of symptom severity over time

${ }^{c}$ For the estimate of median change, negative estimates indicate that function worsened over time, whereas positive estimates indicate that function improved over time

${ }^{\mathrm{d}} n=12$

* Statistically significant $(p<0.05)$ change from baseline to end of study 
experienced significant improvements in worst CIPN pain $(p<0.0001)$, sensory CIPN $(p<0.0001)$, motor CIPN severity ( $p=0.0004)$, physical function $(p=0.003)$, and pain interference $(p=0.002)$ from baseline to the 8 -week follow-up, but the improvements were not statistically significant relative to the control group $(p>0.05)$. Yoga group participants experienced statistically significant improvements in depression $(p=0.04)$ and fatigue $(p=0.05)$ relative to control group participants. Yoga group participants also experienced improvements in sleep-related impairment $(p=0.10)$ and anxiety $(p=0.13)$, relative to controls, although this did not meet statistical significance.

\section{Virtual versus in-person yoga}

Table 3 describes changes in CIPN and co-occurring symptoms over the 8-week intervention period among cancer survivors with chronically painful CIPN who participated in the virtual $(n=15)$ or in-person $(n=6)$ yoga formats. Two patients from the overall yoga sample were removed from these analyses because they were on study at the onset of the COVID-19 pandemic and participated in both the inperson and virtual yoga formats. There were no statistically significant differences in mean change scores in CIPN or cooccurring symptoms between the in-person or virtual yoga intervention participants.

Table 3 Median (range) scores for patient-reported outcomes by yoga format (virtual yoga, $n=15$; in-person yoga, $n=6$ )

\begin{tabular}{|c|c|c|c|c|c|}
\hline \multirow[t]{2}{*}{ Outcomes } & \multicolumn{2}{|l|}{ Median $(\text { range })^{\mathrm{a}}$} & \multicolumn{2}{|c|}{ Estimate of median change (range) } & \multirow{2}{*}{$\begin{array}{l}\text { Comparison of } \\
\text { change between } \\
\text { groups }(p)\end{array}$} \\
\hline & In-person & Virtual & In-person & Virtual & \\
\hline \multicolumn{6}{|l|}{ Worst CIPN pain ${ }^{\mathrm{b}}$} \\
\hline Baseline & $5.3(3.0-9.1)$ & $5.7(3.6-8.1)$ & $-1.2(-4.3-0.6)$ & $-1.7(-7.1$ to 0.4$)$ & 0.61 \\
\hline Eight weeks & $3.9(1.0-8.1)$ & $4.0(0-5.6)$ & & & \\
\hline \multicolumn{6}{|l|}{ CIPN sensory ${ }^{b}$} \\
\hline Baseline & $62.7(25.9-77.8)$ & $44.4(14.8-81.5)$ & $-13.0(-29.6$ to 3.7$)$ & $-11.1(-40.7$ to 11.1$)$ & 0.72 \\
\hline Eight weeks & $37.0(11.1-66.7)$ & $29.6(7.4-88.9)$ & & & \\
\hline \multicolumn{6}{|l|}{ CIPN motor ${ }^{b}$} \\
\hline Baseline & $31.3(12.5-71.4)$ & $29.2(0-70.8)$ & $-13.7(-33.3$ to 33.3$)$ & $-8.3(-39.9$ to 8.3$)$ & 0.69 \\
\hline Eight weeks & $22.9(8.3-52.4)$ & $20.8(0-58.3)$ & & & \\
\hline \multicolumn{6}{|l|}{ Pain interference ${ }^{\mathrm{b}}$} \\
\hline Baseline & $58.5(49.6-63.8)$ & $61.2(41.6-69.7)$ & $-5.8(-19.6$ to 11.2$)$ & $-4.6(-22.2$ to 8.0$)$ & 0.78 \\
\hline Eight weeks & $53.0(41.6-69.7)$ & $53.9(41.6-63.8)$ & & & \\
\hline \multicolumn{6}{|l|}{ Physical function ${ }^{\mathrm{c}}$} \\
\hline Baseline & $39.9(35.6-45.5)$ & $43.5(33.2-57.0)$ & $4.3(-7.6$ to 9.1$)$ & $3.5(-4.3$ to 19.1$)$ & 0.66 \\
\hline Eight weeks & $43.0(35.6-48.3)$ & $48.3(31.9-57.0)$ & & & \\
\hline \multicolumn{6}{|l|}{ Anxiety ${ }^{b}$} \\
\hline Baseline & $57.7(51.2-63.4)$ & $57.7(40.3-71.2)$ & $-2.0(-10.9$ to 10.2$)$ & $-6.5(-17.4$ to 3.9$)$ & 0.21 \\
\hline Eight weeks & $57.6(40.3-63.4)$ & $40.3(40.3-71.2)$ & & & \\
\hline \multicolumn{6}{|l|}{ Depression $^{\mathrm{b}}$} \\
\hline Baseline & $53.2(41-63.9)$ & $53.9(41-63.9)$ & $0(-8.3$ to 4.9$)$ & $0(-12.9$ to 1.7$)$ & 0.92 \\
\hline Eight weeks & $53.9(41-57.3)$ & $49.0(41.0-63.9)$ & & & \\
\hline \multicolumn{6}{|c|}{ Sleep impairment ${ }^{\mathrm{b}}$} \\
\hline Baseline & $58.2(38.7-62.3)$ & $58.2(35.2-66.3)$ & $-5.7(-13.4$ to 24.6$)$ & $-2.2(-21.6$ to 7.1$)$ & 0.99 \\
\hline Eight weeks & $55.6(43.6-63.3)$ & $50.3(30.0-69.5)$ & & & \\
\hline \multicolumn{6}{|l|}{ Fatigue $^{\mathrm{b}}$} \\
\hline Baseline & $56.1(33.7-64.6)$ & $55.1(43.1-75.8)$ & $-7.8(-13.6$ to 21.4$)$ & $-5.5(-14.9$ to 6.0$)$ & 0.45 \\
\hline Eight weeks & $49.8(46-55.1)$ & $48.6(33.7-66.7)$ & & & \\
\hline
\end{tabular}

Note:

${ }^{a}$ Increasing scores for the specified variables indicate worse symptoms, except for physical function, where higher scores represent greater function

${ }^{b}$ For the estimate of median change, negative estimates indicate improvements in symptom severity, whereas positive estimates indicate worsening of symptom severity over time

${ }^{\mathrm{c}}$ For the estimate of median change, negative estimates indicate that function worsened over time, whereas positive estimates indicate that function improved over time 


\section{Discussion}

This pilot study demonstrated the feasibility of a yoga intervention in a group of cancer survivors with chronic CIPN pain, as well as potential benefits for patient-reported outcomes in this population. Participants were more willing to participate in a virtually delivered yoga intervention as accrual and adherence to the virtually delivered yoga intervention were greater than accrual and adherence to in-person yoga. Seemingly, a major reason for participants willingness to prefer virtually delivered yoga is that it addressed travel barriers associated with in-person integrative oncology treatment delivery [29]. For example, the Zakim Center transitioned all clinical integrative oncology programming from in-person to virtual (i.e., livestreamed or on demand) in response to the onset of the COVID-19 pandemic and found that attendance increased by $\sim 647 \%$ during the first 6 months of the COVID-19 pandemic (virtual offerings) in comparison to the 6 months prior to the onset of the COVID19 pandemic (in-person offerings) [30]. The virtual yoga intervention tested in this study is scalable, making it ideal for future studies among diverse populations, including rural residents, who may face travel barriers to oncology symptom management care.

Yoga participants, but not control group participants, experienced improvements in anxiety and fatigue that were within or exceeded the range of published minimally important differences (MID) for the PROMIS ${ }^{\circledR}$ anxiety (MID range $=3.0-4.5)$ and fatigue (3.0-5.0) short forms in patients with cancer [31]. Yoga group participants' improvements in physical function neared the MID (MID range $=4.0-6.0$ ) [31]. Changes in sleep-related impairment were also of significant magnitude, but there are no published data regarding MID for score changes. Although the small numbers reduced power for these comparisons, there were no significant differences in changes in CIPN or co-occurring symptoms between participants engaging in the yoga program virtually or in-person.

Our findings can be compared with results reported by Bao et al. (2020), who randomized 41 breast or gynecological cancer survivors with chronic CIPN symptoms to an 8 -week yoga intervention or usual care. In this study, which combined in-person and at-home practice 7 days per week, yoga group participants experienced a 1.95-point reduction in CIPN pain intensity, but the change was not significant in comparison to the control group $(p=0.14)$ [32]. Our study demonstrated significant within-group changes in sensory CIPN, but we did not observe statistically significant differences between arms like Bao et al. $(p=0.035)$ [32]. In aggregate, our study and the study by Bao and colleagues [32] suggest that yoga may be a promising treatment for cancer survivors with chronic CIPN pain, but a number of unanswered questions remain, including determining the optimal yoga dose and selecting an appropriate active control comparison [33].

\section{Implications for practice}

Currently, painful CIPN is mainly managed through pharmacologic treatment (e.g., pregabalin, duloxetine, or gabapentin) [34]. The use of yoga for painful CIPN management is relevant to oncology clinicians at the point of care as such individuals are in prime positions to refer patients to nonpharmacological interventions as part of comprehensive supportive care for cancer treatment-related symptoms. Based on preliminary evidence suggesting that yoga appears to be a safe and feasible intervention for cancer survivors with chronic CIPN pain, oncology clinicians may consider referring patients to yoga for chronic CIPN pain management. Additional work will be needed to determine the efficacy of yoga for painful CIPN before yoga can be widely recommended as a treatment for chronic CIPN pain.

\section{Limitations}

There are several limitations to this pilot study. The study was conducted in a mainly female, Caucasian, non-Hispanic patient population at one academic medical center, thus decreasing the external generalizability of the study findings. There are several potential threats to the internal validity of the study. The study was designed as a pilot feasibility trial and thus was not powered to detect between-group changes (i.e., yoga vs. control; in-person vs. virtual yoga) in patientreported outcomes. Additionally, participants' symptom severity and adherence to the yoga intervention throughout the study were determined based on self-report alone. Participants and study staff were not blinded to study group assignment and participants in both groups were not provided matched attention (i.e., research staff only interacted with yoga group participants during the study). Assignment to the in-person and virtual yoga groups was driven by timing of enrollment in relation to the COVID-19 pandemic and not through randomization. This could introduce potential bias; for example, it is possible that cancer survivors in the in-person yoga format had disease or demographic characteristics different from those in the virtual yoga format. Finally, the intervention format was altered during the study period due to the COVID-19 pandemic, which complicates the interpretation of the impact of yoga on symptom outcomes.

\section{Conclusion}

The results of this pilot study demonstrated that implementation of a yoga intervention was feasible for cancer survivors 
with chronic CIPN pain. Despite the small sample size, yoga led to statistically and/or clinically significant improvements in several cancer treatment-related symptoms (i.e., depression, fatigue, sleep-impairment, anxiety) among cancer survivors with chronic CIPN pain. Virtually delivered yoga demonstrated high levels of participant engagement. Exploratory analyses suggested similar effects of virtually delivered and in-person yoga, but comparisons were underpowered and unplanned. Given the potential of virtually delivered yoga to facilitate dissemination of yoga to large groups of cancer survivors with CIPN, this approach warrants future research to determine the efficacy of virtual yoga to improve CIPN and co-occurring symptom severity among cancer survivors in an adequately powered, randomized controlled trial. The identification of an efficacious and accessible non-pharmacological treatment for chronic CIPN pain is essential to provide cancer survivors with an alternative to pharmacological treatments to decrease CIPN symptom severity, bolster quality of life, improve oncology symptom management access, and reduce morbidity in the thousands of cancer patients treated with taxanes and platinums each year.

Supplementary Information The online version contains supplementary material available at https://doi.org/10.1007/s11764-021-01081-z.

Acknowledgements The authors would like to acknowledge Erica Fox, Raymond Lamothe, Barbara Halpenny, and Anna Tanasijevic for their assistance with project administration.

Author contribution RK was responsible for the design and conduct of the study, interpretation of the data, and writing of the manuscript. AGH conducted the statistical analyses. JL and AGH contributed to the interpretation of the data. $\mathrm{AGH}, \mathrm{JB}, \mathrm{DB}, \mathrm{JM}, \mathrm{AW}$, and JL were all involved in the design and conception of the study and contributed to the writing of the manuscript. All authors have read and approved the final manuscript.

Funding Oncology Nursing Foundation Research Grant awarded to Robert Knoerl, PhD, RN

Data availability The datasets generated during and/or analyzed during the current study are available from the corresponding author on reasonable request.

Code availability Not applicable.

\section{Declarations}

Ethics approval All procedures performed in studies involving human participants were in accordance with the ethical standards of the institutional and/or national research committee and with the 1964 Helsinki Declaration and its later amendments or comparable ethical standards. The study was approved by the Dana-Farber/Harvard Cancer Center Office for Human Research Studies (18-578).

Consent to participate Informed consent was obtained from all individual participants included in this study.
Consent for publication Not applicable.

Conflict of interest RK has received personal fees (consulting) from Strategy Inc, Spark Healthcare, and System Analytic; and serves on the scientific advisory board of Wellium. JM has received institutional research funding from Boston Biomedical, has served as an advisor/ consultant to COTA Healthcare, and has served on a grant review panel for the National Comprehensive Cancer Network funded by Taiho Pharmaceutical.

\section{References}

1. Ventzel L, Jensen AB, Jensen AR, Jensen TS, Finnerup NB. Chemotherapy-induced pain and neuropathy: a prospective study in patients treated with adjuvant oxaliplatin or docetaxel. Pain. 2016;157:560-8.

2. Winters-Stone KM, Horak F, Jacobs PG, Trubowitz P, Dieckmann NF, Stoyles S, et al. Falls, functioning, and disability among women with persistent symptoms of chemotherapy-induced peripheral neuropathy. J Clin Oncol. 2017;35:2604-12.

3. Kolb NA, Smith AG, Singleton JR, Beck SL, Stoddard GJ, Brown $\mathrm{S}$, et al. The Association of chemotherapy-induced peripheral neuropathy symptoms and the risk of falling. JAMA Neurol. 2016;73:860.

4. Beijers A, Mols F, Dercksen W, Driessen C, Vreugdenhil G. Chemotherapy-induced peripheral neuropathy and impact on quality of life 6 months after treatment with chemotherapy. J community Support Oncol. 2014;12:401-6.

5. Zanville NR, Nudelman KNH, Smith DJ, Von Ah D, McDonald BC, Champion VL, et al. Evaluating the impact of chemotherapyinduced peripheral neuropathy symptoms (CIPN-sx) on perceived ability to work in breast cancer survivors during the first year post-treatment. Support Care Cancer Springer. 2016;24:4779-89.

6. Knoerl R, Chornoby Z, Smith EML. Corrigendum to 'Estimating the frequency, severity, and clustering of SPADE symptoms in chronic painful chemotherapy-induced peripheral neuropathy' Pain Management Nursing 2018;19(4):354-365. Pain Manag Nurs. 2019;20:88.

7. Knoerl R, Chornoby Z, Smith EML. Estimating the frequency, severity, and clustering of SPADE symptoms in chronic painful chemotherapy-induced peripheral neuropathy. Pain Manag Nurs Elsevier. 2018;19:354-65.

8. Smith EM, Pang H, Cirrincione C, Fleishman S, Paskett ED, Ahles T, et al. Effect of duloxetine on pain, function, and quality of life among patients with chemotherapy-induced painful peripheral neuropathy: a randomized clinical trial. JAMA. 2013;309:1359-67.

9. Loprinzi CL, Lacchetti C, Bleeker J, et al. Prevention and Management of Chemotherapy-Induced Peripheral Neuropathy in Survivors of Adult Cancers: ASCO Guideline Update. J Clin Oncol. 2020;38(28):3325-48. https://doi.org/10.1200/JCO.20.01399

10 Knoerl R, Bridges C, Smith G, Yang J, Kanzawa-Lee G, Smith E. Chemotherapy-induced peripheral neuropathy: use of an electronic care planning system to improve adherence to recommended assessment and management practices. Clin J Oncol Nurs. 2018;22:E134-40.

11. Lin NU, Bichkoff $\mathrm{H}$, Hassett MJ. Increasing burden of prior authorizations in the delivery of oncology care in the United States. J Oncol Pract. 2018;14:525-8.

12. Shah A, Hoffman EM, Mauermann ML, et al. Incidence and disease burden of chemotherapy-induced peripheral neuropathy in a population-based cohort. J Neurol Neurosurg Psychiatry. 2018;89(6):636-41. https://doi.org/10.1136/jnnp-2017-317215 
13. Wieland LS, Skoetz N, Pilkington K, Vempati R, D'Adamo CR, Berman BM. Yoga treatment for chronic non-specific low back pain. Cochrane Database Syst Rev. 2017;1(1):CD010671. https:// doi.org/10.1002/14651858.CD010671.pub2

14. Cramer H, Lauche R, Klose P, Lange S, Langhorst J, Dobos GJ. Yoga for improving health-related quality of life, mental health and cancer-related symptoms in women diagnosed with breast cancer. Cochrane Database Syst Rev. 2017;1(1):CD010802. https://doi.org/10.1002/14651858.CD010802.pub2

15. Danhauer SC, Addington EL, Cohen L, et al. Yoga for symptom management in oncology: A review of the evidence base and future directions for research. Cancer. 2019;125(12):1979-89. https://doi.org/10.1002/cncr.31979

16. Dworkin RH, Turk DC, Peirce-Sandner S, et al. Research design considerations for confirmatory chronic pain clinical trials: IMMPACT recommendations. Pain. 2010;149(2):177-93. https:// doi.org/10.1016/j.pain.2010.02.018

17. Dworkin RH, Turk DC, Farrar JT, Haythornthwaite JA, Jensen MP, Katz NP, et al. Core outcome measures for chronic pain clinical trials: IMMPACT recommendations. Pain. 2005;113:9-19.

18. Cleeland CS, Ryan KM. Pain assessment: global use of the Brief Pain Inventory. Ann Acad Med Singapore. 1994;23:129-38.

19. Cella D, Yount S, Rothrock N, Gershon R, Cook K, Reeve B, et al. The Patient-Reported Outcomes Measurement Information System (PROMIS): progress of an NIH Roadmap cooperative group during its first two years. Med Care NIH Public Access. 2007;45:S3-11.

20. Amtmann D, Cook KF, Jensen MP, Chen W-H, Choi S, Revicki $\mathrm{D}$, et al. Development of a PROMIS item bank to measure pain interference. Pain. 2010;150:173-82.

21. Yu L, Buysse DJ, Germain A, Moul DE, Stover A, Dodds NE, et al. Development of short forms from the PROMIS ${ }^{\mathrm{TM}}$ Sleep Disturbance and Sleep-Related Impairment Item Banks. Behav Sleep Med. 2012;10:6-24.

22. Kroenke K, Yu Z, Wu J, Kean J, Monahan PO. Operating characteristics of PROMIS four-item depression and anxiety scales in primary care patients with chronic pain. Pain Med. 2014;15:1892-901.

23. Bartlett SJ, Orbai A-M, Duncan T, DeLeon E, Ruffing V, CleggSmith K, et al. Reliability and validity of selected PROMIS measures in people with rheumatoid arthritis. Zhang C, editor. PLoS One. 2015;10:e0138543.

24. Jensen RE, Potosky AL, Reeve BB, Hahn E, Cella D, Fries J, et al. Validation of the PROMIS physical function measures in a diverse US population-based cohort of cancer patients. Qual Life Res NIH Public Access. 2015;24:2333-44.

25. Postma TJ, Aaronson NK, Heimans JJ, Muller MJ, Hildebrand JG, Delattre JY, et al. The development of an EORTC quality of life questionnaire to assess chemotherapy-induced peripheral neuropathy: the QLQ-CIPN20. Eur J Cancer. 2005;41:1135-9.

26. Smith EML, Barton DL, Qin R, Steen PD, Aaronson NK, Loprinzi CL. Assessing patient-reported peripheral neuropathy: the reliability and validity of the European Organization for Research and Treatment of Cancer QLQ-CIPN20 Questionnaire. Qual Life Res. 2013;22:2787-99.

27. Lavoie Smith EM, Haupt R, Kelly JP, Lee D, Kanzawa-Lee G, Knoerl R, et al. The content validity of a chemotherapy-induced peripheral neuropathy patient-reported outcome measure. Oncol Nurs Forum. 2017;44:580-8.

28 Moher D, Schulz KF, Altman D. The CONSORT statement: revised recommendations for improving the quality of reports of parallel-group randomized trials. J Am Med Assoc. 2001;285:1987-91.

29. Latte-Naor S, Mao JJ. Putting integrative oncology into practice: concepts and approaches. J Oncol Pract. 2019;15(1):7-14. https:// doi.org/10.1200/JOP.18.00554

30. Knoerl R, Phillips CS, Berfield J, et al. Lessons learned from the delivery of virtual integrative oncology interventions in clinical practice and research during the COVID-19 pandemic. Support Care Cancer. 2021;29(8):4191-4. https://doi.org/10.1007/ s00520-021-06174-0

31. Yost KJ, Eton DT, Garcia SF, Cella D. Minimally important differences were estimated for six Patient-Reported Outcomes Measurement Information System-Cancer scales in advanced-stage cancer patients. J Clin Epidemiol. 2011;64:507-16.

32. Bao T, Zhi I, Baser R, et al. Yoga for chemotherapy-induced peripheral neuropathy and fall risk: a randomized controlledtrial. JNCI Cancer Spectr. 2020;4(6):pkaa048. https://doi.org/10.1093/ jncics/pkaa048

33. Park CL, Groessl E, Maiya M, Sarkin A, Eisen S V., Riley K, et al. Comparison groups in yoga research: a systematic review and critical evaluation of the literature. Complement. Ther. Med. Churchill Livingstone; 2014 [cited 2021 Feb 8]. p. 920-9.

34. Selvy M, Pereira B, Kerckhove N, et al. Prevention, diagnosis and management of chemotherapy-induced peripheral neuropathy: a cross-sectional study of French oncologists' professional practices. Support Care Cancer. 2021;29(7):4033-43. https://doi.org/ 10.1007/s00520-020-05928-6

Publisher's note Springer Nature remains neutral with regard to jurisdictional claims in published maps and institutional affiliations. 